\title{
Psicooncología
}

ISSN: 1696-7240

\section{Conviviendo con la Neurofibromatosis tipo 1: Revisión de la literatura}

\author{
Nancy Castrillón ${ }^{1, *} ;$ Ignacio Blanco ${ }^{2}$
}

Recibido: 10 de enero de 2018 / Aceptado: 24 de febrero de 2018

Resumen. Objetivo: En este estudio se realiza una revisión de la literatura sobre el impacto psicológico y emocional que conlleva la Neurofibromatosis tipo 1 (NF1) en los pacientes adultos. Método: Se realizó una revisión bibliográfica en PubMed (Medline) entre enero del 2007 y abril del 2017 de artículos publicados en el idioma inglés. Se encontraron un total de 75 artículos de los cuales se seleccionaron 23 con base en los criterios de inclusión y exclusión definidos para esta revisión. Resultados: Prácticamente todos los estudios vienen a concluir que los cambios y/o desfiguración en la imagen corporal o apariencia física, la visibilidad, el dolor y la gravedad de la enfermedad son el eje central sobre el cual gira el deterioro en la calidad de vida de las personas con NF1, debido a las dificultades que tienen los enfermos para adaptarse a los cambios físicos y para hacer frente a los problemas de ansiedad y el estrés psicológico que genera la carga de la enfermedad. Conclusiones: Conocer el impacto psicológico y emocional que tiene la NF1 permitirá desarrollar e implementar intervenciones psicoterapéuticas y psicosociales específicas para este grupo de población con el objetivo de facilitarles que puedan afrontar las consecuencias y los retos que trae asociada la enfermedad.

Palabras clave: Neurofibromatosis tipo 1; calidad de vida; imagen corporal; ansiedad; estrés psicológico; impacto emocional.

\section{[en] Living with Neurofibromatosis Type 1: Review of the literature}

\begin{abstract}
Objective: This study was done to conduct a review of the literature on the psychological and emotional impact of NF1 in adult patients. Method: A literature review in PubMed (Medline) of articles published in the English languaje was conducted between January 2007 and April 2017. A total of 75 articles were found, of which 23 were selected based on the inclusion and exclusion criteria defined for this review. Results: Almost all studies conclude that changes and/or disfigurement in the corporal image or physical appearance, the visibility, the pain and the severity of the disease are the central axes on which the deterioration in the quality of life of the people with NF1; due to the difficulties that patients have to adapt to the physical changes and to cope the problems of anxiety and psychological stress generated by the burden of the disease. Conclusions: Knowing the psychological and emotional impact of NF1 will allow the development and implementation of specific psychotherapeutic and psychosocial interventions for this population group with the aim of facilitating them to face the consequences and challenges associated with the disease.
\end{abstract}

Keywords: Neurofibromatosis type 1; quality of life; body image; anxiety; psychological stress; emotional impact.

1 Tecnocampus Parc. TecnoCampus Mataró-Maresme. Carrer d'Ernest Lluch, 32, 08302 Mataró, Barcelona E-mail: mcastrillon@edu.tecnocampus.cat; mnancycastrillon@gmail.com

2 Ignacio Blanco. Programa de Asesoramiento y Genética Clínica. Institut Català de la Salut. Hospital Universitari Germans Trias i Pujol.

E-mail: iblanco.germanstrias@gencat.cat

* Dirección de correspondencia: Nancy Castrillón. Tecnocampus Parc. TecnoCampus Mataró-Maresme. Carrer d'Ernest Lluch, 32, 08302 Mataró, Barcelona.

E-mail: mcastrillon@edu.tecnocampus.cat; mnancycastrillon@gmail.com 
Sumario. 1. Introducción 2. Material y métodos 3. Resultados 4. Discusión 5. Conflicto de intereses 6. Referencias bibliográficas.

Cómo citar: Castrillón N, Blanco I. Conviviendo con la Neurofibromatosis tipo 1: Revisión de la literatura. Psicooncología 2018;15:37-48. Doi: 10.5209/PSIC.59173.

\section{Introducción}

La neurofibromatosis (NF) comprende un grupo de desórdenes neurogenéticos crónicos y progresivos distintos, como: la Neurofibromatosis tipo 1 (NF1), la neurofibromatosis tipo 2 (NF2) y la Schwannomatosis; los cuales se transmiten de manera autosómica dominante ${ }^{(1)}$; sin embargo, algunos casos nuevos surgen de forma espontánea debido a una mutación "de novo" en los genes del individuo ${ }^{(2)}$. De esta triada de desórdenes genéticos, la NF1 es el trastorno autosómico dominante hereditario más frecuente, con una prevalencia estimada de 1:3000 casos por nacimiento; afecta a ambos sexos y a todos los grupos raciales; no obstante, diversos estudios epidemiológicos han encontrado que es más frecuente en poblaciones de ascendencia europea ${ }^{(1,2)}$.

La NF1 es causada por mutaciones puntuales en el gen supresor tumoral neurofibromina $1 \mathrm{NF1}$ (17q11.2) y raramente (5\%) se presenta por deleción completa o incompleta del gen NF1. Se calcula que aproximadamente entre el 30 y el $50 \%$ de los casos nuevos no tienen antecedentes familiares, sino que surgen como una nueva mutación genética espontánea del gen $N F l^{(1,3)}$.

Las manifestaciones clínicas de la NF1 pueden darse desde el nacimiento o poco después; sin embargo, los signos de la enfermedad casi siempre se hacen evidentes a los 10 años $^{(3)}$ y estos van aumentando con la edad. El diagnóstico clínico de la enfermedad se realiza con base a "los criterios diagnósticos" The National Institutes of Health (NIH), que se definieron en el año 1987 en la conferencia de desarrollo de consenso sobre neurofibromatosis. Actualmente, no existe una cura para la enfermedad y, a pesar de que su pronóstico es bueno, existe una alta morbilidad debido su carácter variable e impredecible ${ }^{(4)}$ y las complicaciones asociadas, como: hipertensión, tumores gastrointestinales, anomalías óseas, dolor y síntomas neurológicos ${ }^{(5)}$.

El sello distintivo de la NF1 son los neurofibromas cutáneos o tumores benignos de la piel, los neurofibromas plexiformes difusos que crecen a lo largo de un nervio, las efélides o pecas en la ingle y las axilas y las manchas en la piel de color café con leche ${ }^{(1,3)}$; las cuales, son la primera manifestación de la enfermedad y están presentes en el $100 \%$ de los niños.

Los neurofibromas cutáneos están presentes en un 30\% de los enfermos $\mathrm{y}$, generalmente se manifiestan en la adolescencia, y continúan apareciendo y desarrollándose a lo largo de la edad adulta ${ }^{(1)}$. Por su parte, los neurofibromas plexiformes internos están presentes en un $20 \%$ de los individuos, pero el número y la cantidad que pueden llegar a desarrollarse, además de que varían en cada individuo, también generan diversas complicaciones, ya que pueden causar desfiguración, dolor y problemas funcionales como parestesias o debilidad ${ }^{(1,3)}$. Así mismo, existe un riesgo de por vida de alrededor del $10 \%$ y el $12 \%$ de que se conviertan en malignos y que 
desarrollen cáncer, principalmente, entre los 20-40 años de edad ${ }^{(4)}$. Aunque, algunos tumores pueden tratarse con cirugía, radiación y quimioterapia, la enfermedad lleva asociada una reducción en la esperanza de vida de los enfermos ${ }^{(6)}$.

Otros síntomas que caracterizan a la enfermedad es la presencia de gliomas de las vías ópticas, los cuales generalmente se desarrollan antes de los 6 años y rara vez progresan; los nódulos de Lisch o hamartomas del iris, macrocefalia, escoliosis y anomalías óseas como pseudoartrosis o falsa articulación ${ }^{(1,3)}$ y alrededor de un tercio de la población afectada presenta baja estatura ${ }^{(5)}$.

En los niños y adolescentes se ha encontrado un ligero deterioro mental, déficits cognitivos, problemas de comportamiento, trastorno de déficit de atención con hiperactividad (TDHA) $)^{(3,5)}$, soledad, retraimiento ${ }^{(5)}$, dificultades en el funcionamiento ejecutivo y problemas de aprendizaje ${ }^{(7)}$. En relación con lo anterior, un estudio publicado en el año 2014 que valoro el rendimiento cognitivo de un pequeño grupo de personas de edad avanzada, concluyó que los déficits cognitivos como: las habilidades visuoespaciales, la memoria, la función ejecutiva y los problemas de atención no mejoraban con la edad; por lo que el deterioro cognitivo es una característica común de la NF1 que puede permanecer estable en toda la vida útil de algunos enfermos ${ }^{(8)}$.

En esencia, la NF1 tiene un carácter variable e impredecible que no le permite a los individuos que la padecen ejercer ningún tipo de control sobre su evolución y desarrollo; y sobre las desfiguraciones visibles que esta genera en la imagen corporal debido a la aparición de los neurofibromas ${ }^{(2)}$ con todas las consecuencias emocionales, psicológicas, familiares, sociales y relacionales que esto conlleva para la vida de los enfermos. Es por ello que, es importante conocer los estudios que se han realizado al respecto, ya que se ha demostrado que los individuos que sufren una enfermedad que incluye cambios visibles en su apariencia corporal como desfiguraciones, suelen presentar un mayor grado de trastornos psicológicos que la media de la población ${ }^{(9,10)}$; los cuales, a menudo surgen como una reacción a la desfiguración, al estigma social percibido y a los cambios negativos en el estilo de vida ${ }^{(11)}$. El objetivo principal de esta revisión es conocer el impacto psicológico y emocional que tiene la NF1 en los adultos, ya que esto permitirá desarrollar e implementar intervenciones psicoterapéuticas y psicosociales específicas para este grupo de población con el objetivo de facilitarles que puedan afrontar las consecuencias y los retos que trae asociada la enfermedad.

\section{Material y métodos}

Antes de hacer la revisión de la literatura, se realizó una identificación de todas aquellas palabras clave que podrían estar relacionadas con el impacto personal de la enfermedad; posteriormente se procedió a realizar una búsqueda de términos $\mathrm{MeSH}$ de dichas palabras para ver si había correspondencia o si era necesario reemplazarlas por otras que se ajustarán al concepto inicial. En total se definieron 23 palabras claves, de las cuales quedaron 9: Calidad de vida o estilo de vida, adaptación psicológica o ajuste emocional, imagen corporal, auto-concepto, autoestima, estigma social, relaciones interpersonales, estrés psicológico y costo de la enfermedad. La definición de los descriptores para realizar la búsqueda se muestra en la tabla 1. 
Se realizó una búsqueda en PubMed (Medline). Se revisaron artículos en inglés publicados entre enero del 2007 y abril del 2017, llevados a cabo en pacientes mayores de 19 años, y se descartaron estudios biomédicos y genéticos, estudios de neuroanatomía y neuroimagen y estudios relacionados con la asistencia hospitalaria.

Tabla 1. Definición de los descriptores de búsqueda

\begin{tabular}{|c|c|c|}
\hline Conceptos & Reemplazado por & $\begin{array}{l}\text { Termino MeSH para } \\
\text { realizar la búsqueda }\end{array}$ \\
\hline Quality life & Quality of Life/Life Style & Quality of Life/Life Style \\
\hline Body Image & No & Body Image \\
\hline Social Stigma & No & Social Stigma \\
\hline Self-Concept & No & Self-Concept \\
\hline Self-Steem & Self-Assessment & Self-Assessment \\
\hline Self-Confidence & Gestalt Therapy & No \\
\hline Self-Perception & No & No \\
\hline Self-Image & Self-concept & No \\
\hline Self-Consciousness & No & No \\
\hline Social Interactions & Interpersonal relations & Interpersonal relations \\
\hline Emotions & Psychological factors & No \\
\hline Feelings & Psychological factors & No \\
\hline $\begin{array}{l}\text { Adaptation Psycholo- } \\
\text { gical }\end{array}$ & No & No \\
\hline Psychological distress & Psychological factors & No \\
\hline Psychological burden & Cost of illness & Cost of Illness \\
\hline Emotional Adjustment & Adaptation psychological & $\begin{array}{l}\text { Adaptation psychological } \\
\text { OR emotional adjustment }\end{array}$ \\
\hline Ways coping & Adaptation psychological & No \\
\hline Skill coping & Adaptation psychological & No \\
\hline Sickness impact & Quality life & No \\
\hline Emotional impact & Stress Psychological/psychology & $\begin{array}{l}\text { Stress Psychological/ } \\
\text { Psychology }\end{array}$ \\
\hline
\end{tabular}

\section{Resultados}

Se encontraron un total de 75 artículos en la base de datos consultada, de los cuales sólo 23 cumplieron con los criterios de elegibilidad establecidos para esta revisión, como se muestra en la tabla 2 . Se destaca que, la mayoría de los estudios 
que se han realizado sobre la NF1 y su relación con la calidad de vida y la imagen corporal, han sido llevados a cabo en países anglosajones o de habla inglesa.

Tabla 2. Estrategia de búsqueda

\begin{tabular}{llc}
\hline \multicolumn{1}{c}{ Criterios de inclusión } & \multicolumn{1}{c}{ Descriptores } \\
\hline Artículos en Inglés & Quality of Life OR Style Life & 20 \\
NF1/Psichology & Interpersonal relations & 7 \\
Adult (Age 19+) & Adaptation Psychological OR Emotional Adjustment & 5 \\
& Self Concept & 6 \\
& Stress Psychological/psychology & 2 \\
& Body Image & 2 \\
& Self Assesment & 0 \\
& Cost of Illness & 0 \\
& Social Stigma & 0 \\
\hline
\end{tabular}

\begin{tabular}{ll}
\hline 33 & 42 \\
\hline & TOTAL $=75$ \\
\hline
\end{tabular}

\section{Artículos duplicados}

22

Artículos excluidos con base en los criterios de exclusión

30

TOTAL Artículos para la revisión

23

El primer estudio que se publicó sobre los aspectos psicológicos de la NF1 en personas adultas, fue realizado en el año 1995, bajo el título: "Psychological aspects of von Recklinghausen neurofibromatosis (NF1)". En dicho estudio, se hizo una revisión de la literatura existente que había hasta ese momento sobre la enfermedad y su relación con cinco aspectos: Apariencia física, discapacidad intelectual, aspectos neuropsicológicos, problemas de aprendizaje, y desordenes psiquiátricos. Dentro de los resultados que se encontraron, se destaca que los cambios físicos que genera la enfermedad trae como consecuencia que los enfermos tengan una distorsión y una pobre confianza en sí mismos, hasta el punto que llegan aislarse socialmente debido a las dificultades que desarrollan para poder establecer relaciones sociales; además, también encontraron que los individuos con NF1 son propensos a tener dificultades de aprendizaje y a desarrollar diversos tipos de enfermedades mentales como depresión y ansiedad ${ }^{(12)}$. 
Apartir de esta primera revisión, quedó demostrado que la NF1 es una enfermedad que por su misma esencia conlleva un alto riesgo de ejercer una influencia negativa a nivel psicológico y emocional en los enfermos; sin embargo, los estudios que se han hecho sobre la NF1 y su impacto en las personas en la edad adulta, en su gran mayoría se han centrado en valorar y describir el impacto que tiene la enfermedad en la calidad de vida de los enfermos. Encontrándose que prácticamente todos estos estudios vienen a decir que los cambios y/o desfiguración en la imagen corporal o apariencia física, la visibilidad y la gravedad de la enfermedad son el eje central sobre el cual gira el deterioro en la calidad de vida de las personas con NF1 debido a las dificultades que tienen para afrontar la enfermedad y para adaptarse a los cambios físicos que esta genera; para desarrollar estrategias y habilidades que les ayuden a hacer frente a las dificultades sociales y emocionales; para superar las barreras y las dificultades en el momento de establecer relaciones interpersonales y vínculos afectivos estrechos; para hacer frente a los problemas de ansiedad y el estrés psicológico que genera la carga de la enfermedad; para aumentar la autoestima, autoconfianza y autoconcepto; para disminuir los sentimientos de vergüenza y aislamiento social; para superar los sentimientos de soledad y la percepción negativa que tienen de sí mismos y del propio cuerpo, y para afrontar el estigma social ${ }^{(5,6,13-26)}$.

Lo anterior queda sustentado por una revisión sistemática publicada en el 2013, que identifico siete estudios realizados entre el año 2001 y 2013, sobre la calidad de vida de las personas que tienen NF1. Dicha revisión informo que, aunque la diversidad de los instrumentos utilizados en los estudios para medir la calidad de vida hace difícil poder comparar los resultados; la mayoría de los estudios concluyen que las personas con NF1 obtienen unas puntuaciones más bajas en calidad de vida en comparación con la población general. Además, encontraron que los estudios reportaban que la visibilidad y la gravedad de la enfermedad actuaban como fuertes predictores en calidad de vida específicos para la piel; sin embrago, con otros predictores de calidad de vida, los resultados eran débiles o poco concluyentes ${ }^{(14)}$.

En un estudio realizado en Australia por Crawford et al. (2015) sobre el impacto que ejerce la NF1 en la salud y la calidad de la vida de los adultos; también encontraron que la visibilidad y gravedad de la enfermedad generan malestar y preocupación por los cambios cosméticos y la desfiguración que generan los neurofibromas en el cuerpo ${ }^{(5)}$; conllevando que las personas con NF1 construyan una percepción negativa de sus propios cuerpos ${ }^{(5,6,16-19)}$. Este estudio también encontró que los pacientes presentan un alto grado de preocupación por el dolor, la incertidumbre sobre la evolución de la enfermedad, y el riesgo de la trasmisión a la descendencia; destacándose que muchas de las preocupaciones son compartidas tanto por los adultos levemente afectados como por los más severamente afectados ${ }^{(5)}$.

Las percepciones negativas que construyen las personas con NF1 sobre su cuerpo, puede ser explicadas por el hecho de que la NF1 es una enfermedad crónica progresiva e impredecible; por tanto, los pacientes no pueden inhibir o ejercer ningún tipo de control sobre la aparición de los neurofibromas y los cambios físicos, y la desfiguración que estos generan en su cuerpo ${ }^{(5,6,14,20)}$.

Con respecto a los estudios realizados en Europa, se destaca un estudio realizado en Alemania por Granström et al. (2012); dicho estudio analizó por primera vez y 
con medidas estandarizadas como las personas con NF1 experimentan y evalúan su cuerpo; también hicieron una comparación entre pacientes con NF1 y pacientes que padecían psoriasis y anorexia ${ }^{(6)}$. A las conclusiones que llegaron fue que, las mujeres con NF1 se sentían menos atractivas y más inseguras con respecto a su propio cuerpo en comparación con las pacientes que padecían psoriasis. También concluyó que las mujeres con NF1 tienen características similares con respecto a la percepción negativa ${ }^{(6,17,20)}$, restrictiva y distorsionada que tienen de su propio cuerpo las pacientes que sufren anorexia ${ }^{(6)}$. En comparación con los hombres, un estudio realizado en Noruega, encontró que estos presentaban poca preocupación por las manifestaciones visibles de la NF1(16).

De igual forma se ha encontrado que la imagen corporal influye de manera negativa en la vida sexual de las mujeres ${ }^{(6,16,17)}$, debido a que presentan mayor timidez ${ }^{(17)}$ y albergan sentimientos de vergüenza, repulsión y rechazo anticipado como consecuencia de hacer visibles los neurofibromas de su cuerpo en sus relaciones íntimas, comportando que se sientan sexualmente insatisfechas ${ }^{(6)}$.

Con respecto a los trastornos psiquiátricos, Kodra et al. (2009) encontraron que los individuos con NF1 tienen un mayor riesgo de comorbilidad con ciertas manifestaciones psiquiatras como la depresión ${ }^{(18)}$. Aunque, el único estudio que se ha hecho con el objetivo de medir la prevalencia de los síntomas depresivos en adultos con NF1 y su calidad de vida, sugiere que si bien se desconoce si los síntomas depresivos son una manifestación a la NF1 o una reacción secundaria a vivir con la enfermedad, la alta prevalencia de los síntomas depresivos demuestra que la enfermedad genera un gran impacto emocional negativo a nivel personal (22), $\mathrm{y}$ un deterioro en la calidad de vida de los personas con NF1 ${ }^{(23)}$.

A este respecto, Wang et al. (2012), examinaron el funcionamiento emocional entre los pacientes con NF1, NF2 y Schwannomatosis; y concluyeron que si bien las manifestaciones clínicas para cada tipo de síndrome son diferentes; la neurofibromatosis parece estar asociada con una reducción del bienestar emocional en comparación con la población general ${ }^{(21)}$.

De igual manera, Bicudo et al. (2016), realizaron un estudio con el objetivo de analizar como construyen la calidad de vida los adultos con NF1, y el cual fue realizado en América del Sur (Brasil); e indicaron que la visibilidad de la enfermedad es un factor predictivo de riesgo para las dificultades sociales y los problemas a nivel emocional que experimentan los enfermos con $N F 1^{(19)}$; lo que también había sido sugerido por un estudio realizado con 129 pacientes en Italia en el año 2005, y publicado en el año 2009(18).

La mayoría de los estudios informan que la carga o estrés psicológico que conlleva la visibilidad de la NF1, les genera a los pacientes muchas dificultades para poder establecer relaciones sociales ${ }^{(13-19,23-27)}$. Debido a que a menudo tienen que luchar para poder conservar sus vínculos interpersonales ${ }^{(24)}$; pero, aun así este sobre esfuerzo se ve superado por los sentimientos de vergüenza y timidez que padecen $^{(20)}$, por la percepción negativa que tienen de sí mismos y por las pobres habilidades sociales que poseen ${ }^{(24)}$; hasta el punto que muchos estudios corroboran que las personas con NF1 son muy propensas a retirase y aislarse socialmente por la visibilidad de la enfermedad ${ }^{(5,15,16,24,25)}$. La soledad es una experiencia aversiva que afecta el funcionamiento social, afectivo y cognitivo de un individuo y puede, por lo tanto, tener un alto impacto en el perfil psicológico de las personas con NF1 ${ }^{(26)}$. 
En este orden de ideas, Ejerskov et al. compararon las experiencias de soledad asociada a factores como la depresión, la timidez, el acoso y la percepción subjetiva que se tiene de la carga de la enfermedad entre hermanos con NF1 y hermanos no afectados con un rango de edad entre 13-23 años; los resultados encontrados indicaron que los hermanos con NF1presentaban una mayor incidencia y un alto grado de soledad y dificultades para establecer vínculos sociales, en comparación con los hermanos no afectados ${ }^{(26)}$.

Sin embargo, un estudio realizado por Hummelvoll y Antonsen y publicado en el año 2013; informaron que, si bien la visibilidad de la enfermedad contribuye a que los adultos se retiren y se aíslen socialmente, aun así no quedaba muy claro que otros factores podrían estar contribuyendo a las pobres habilidades sociales que suelen presentar las personas con $N F 1^{(16)}$. Como posible respuesta alternativa a este comportamiento; se ha planteado que los déficits sociales pueden estar directamente relacionados con deficiencias en los procesos cognitivos que subyacen al comportamiento social y con los diversos problemas que presentan en la regulación emocional las personas con $\mathrm{NF} 1^{(15,16,24)}$.

Lo anterior, va en la misma línea de otro estudio realizado por Pride et al. $(2014)^{(25)}$, el cual informo que las personas con NF1 presentan alteraciones en su cognición social; es decir, en sus capacidades para recibir y procesar la información social que les llega del mundo externo. Específicamente, se encontró que las personas con NF1 tienen dificultades para poder reconocer las expresiones faciales de ira y miedo, y el sarcasmo paradójico; lo que conlleva a que también tengan dificultades para poder regular su comportamiento y por ende a que fracasen en sus interacciones sociales ${ }^{(25)}$.

Otros estudios refieren que uno de los principales factores que influyen para que las personas con NF1 se depriman ${ }^{(22)}$, para que presenten mayores niveles de estrés psicológico ${ }^{(6,13)}$ y para que se aíslen y se retiren socialmente, es el estigma social que padecen por la visibilidad y la desfiguración que genera la NF1 $1^{(14-19,21)}$. La NF1 es una enfermedad que por su misma esencia fractura la representación mental que se tiene establecida a nivel personal y social de lo que es físicamente normal y anormal; lo que conlleva a que los enfermos se protejan asilándose por la conciencia que tienen de sí mismos ${ }^{(14)}$ y por los efectos que genera la visibilidad de la enfermedad a nivel social ${ }^{(27)}$. A este respecto, Wang D et al. (2012), concluyeron que no había asociación entre la gravedad de la enfermedad y el funcionamiento emocional ${ }^{(21)}$; y sugirieron que en la vida de los pacientes, los factores psicosociales eran más importantes o tan importantes como la gravedad de la enfermedad ${ }^{(19)}$ para determinar cómo experimentan y cómo afrontan los enfermos el tener NF1.

\section{Discusión}

Esta revisión resume el resultado de 23 estudios que han evaluado la calidad de vida de los pacientes y en los cuales se han incluido los aspectos psicológicos y sociales de pacientes adultos afectados por NF1. Prácticamente todos los estudios revisados informan que la NF1 es una enfermedad que conlleva una carga para los enfermos, traducida en un impacto psicológico y un deterioro en su calidad de vida; ya que el curso clínico e impredecible de la enfermedad, las complicaciones médicas, la variabilidad y severidad de los síntomas, los cambios físicos y la estigmatización 
social coloca a los enfermos ante el reto y la constante necesidad de hacer uso de todos los recursos psicológicos, emocionales y mentales que poseen para hacer frente a las características intrínsecas de la enfermedad.

El estigma social, es un constructo implícito en la NF1, en virtud de que los cambios visibles que generan los neurofibromas, quiebran la representación mental que se tiene a nivel personal y social de lo que está establecido como físicamente normal y anormal, lo que supone mayores niveles de estrés psicológico, soledad, depresión y deterioro en la calidad de vida de los enfermos. De hecho muchos enfermos prefieren retirase y aislarse socialmente por los sentimientos de vergüenza, timidez, baja autoestima y bajo autoconcepto que padecen, y por la exclusión y el estigma social al cual se ven sometidos por la visibilidad de los neurofibromas cutáneos. El aislamiento y por ende la ausencia de vínculos sociales y personales estrechos no les permite a los pacientes poder desarrollas habilidades sociales, por lo que muchos se encuentran ante la necesidad de realizar un sobre esfuerzo para poder conservar sus vínculos afectivos; pero, por lo general fracasan en los intentos que hacen debido a los sentimientos de vergüenza y la baja autoestima que poseen. A este respecto, los estudios también sugieren que los déficits sociales pueden estar directamente relacionados con deficiencias en los procesos cognitivos que subyacen al comportamiento social y con los diversos problemas que presentan los pacientes con NF1 para poder regular su comportamiento.

La soledad es un sentimiento generalizado en la NF1, la cual ejerce un alto impacto en el perfil psicológico de los pacientes, ya que afecta su funcionamiento social, afectivo y cognitivo; además la soledad que padecen los pacientes se ha asociado a factores como la timidez, el acoso y la percepción subjetiva que se tiene de la carga de la enfermedad y la depresión. Aunque, hay poca evidencia con respecto a la NF1 y las manifestaciones psiquiatras, se ha encontrado que los pacientes con NF1 tienen un mayor riesgo de morbilidad con la depresión, y a pesar de que no hay claridad sobre si los síntomas depresivos son una manifestación a la NF1 o una reacción secundaria a vivir con la enfermedad, la alta prevalencia de dichos síntomas demuestran que la NF1 acarrea un gran impacto emocional negativo a nivel personal y un deterioro en la calidad de vida de los enfermos.

La NF1 es una enfermedad crónica, progresiva e impredecible que genera en los pacientes altos niveles de preocupación y estrés psicológico, ya que no pueden inhibir o ejercer ningún tipo de control sobre la aparición de los neurofibromas, los cambios físicos y la desfiguración que estos puedan llegar a generan en su cuerpos; por tanto, es muy característico que con el transcurrir del tiempo los pacientes construyan percepciones negativas alrededor de su propio cuerpo, las cuales están entretejidas con una pobre autoestima y una baja autoconfianza. Aunque, hay poca evidencia con respecto al género, se encuentra que la imagen corporal influye negativamente en la vida sexual de las mujeres, en comparación con los hombres, debido a que estas presentan mayor timidez, inseguridad y sentimientos de vergüenza, repulsión y rechazo anticipado en sus encuentros sexuales.

Todos los estudios realizados indican que la NF1 es una enfermedad que afecta todas las áreas de la vida de los pacientes; y los factores psicosociales son más importantes o tan importantes como la gravedad y la variabilidad de la enfermedad para determinar cómo experimentan y cómo afrontan los enfermos el tener NF1. Por tanto, conocer las características de la enfermedad y el impacto emocional, psicológico, mental y social que tiene la NF1 en la vida de los enfermos, permitirá 
poder desarrollar y diseñar intervenciones psicosociales y psicoterapéuticas específicas para este grupo de pacientes, con el objetivo de facilitarles el que puedan afrontar las consecuencias y los desafíos que trae asociada la enfermedad; pero teniendo en cuenta que no existe un concepto de cuerpo único, simple y natural, sino que la representación corporal está construida y delimitada por la suma de una multitud de pequeñas designaciones y prácticas culturas específicas que no permiten ser generalizables de un contexto socio-cultural a otro; al igual que el concepto y la vivencia de la enfermedad debe ser abordada teniendo en cuenta la subjetividad y la individualidad de cada enfermo.

\section{Conflicto de intereses}

Los autores declaran no tener ningún conflicto de intereses.

\section{Referencias bibliográficas}

1. Theos A, Boyd KP, Korf BR. The Neurofibromatoses. In: Irvine AD, Hoeger PH, Yan AC, editors. Harper's Textbook of Pediatric Dermatology, Volume 1, 2, Third Edition. Oxford, UK: Wiley-Blackwell, 2011. doi: 10.1002/9781444345384.ch128.

2. National Institute of Neurological Disorders and Stroke (NINDS). Neurofibromatosis Fact Sheet. 2011. [Acceso 10 de enero de 2018]. Disponible en: /www-ninds-nih-gov. sire.ub.edu/Disorders/All-Disorders/Neurofibromatosis-Information-Page; consultado el 16 de abril del 2017.

3. National Institute of Neurological Disorders and Stroke (NINDS).Neurofibromatosis Fact Sheet. 2011. [Acceso 10 de enero de 2018]. Disponible en: https://espanol-nindsnihgov.sier.ub.edu/trastornos/neurofibromatosis.htm; consultado el 16 de abril del 2017.

4. Neurofibromatosis type 1. Orphanet Enciclopedia 2014. [Acceso 10 de enero de 2018]. Disponible en: http://www.orpha.net.net; consultado el 20 de abril del 2017.

5. Crawford HA, Barton B, Wilson MJ, Berman Y, McKelvey-Martin VJ, Morrison PJ, et al. The impact of Neurofibromatosis Type 1 on the health and wellbeing of Australian adults. J Genet Couns 2015; 24:931-44. doi:10.1007/s10897-015-9829-5.

6. Granström S, Langenbruch A, Augustin M, Mautner V-F. Psychological burden in adult neurofibromatosis Type 1 patients: Impact of disease visibility on body image. Dermatology 2012; 224: 160-7. doi:10.1159/000337548.

7. Barke J, Harcourt D, Coad J. "It"s like a bag of pick and mix - you don't know what you are going to get': young people's experience of neurofibromatosis Type 1. J Adv Nurs 2014; 70: 1594-603. doi:10.1111/jan.12319.

8. Costa D de S, de Paula JJ, de Rezende NA, Rodrigues LOC, Malloy-Diniz LF, RomanoSilva MA, et al. Neuropsychological impairments in elderly Neurofibromatosis type 1 patients. Eur J Med Genet 2014; 57: 216-9. doi:10.1016/j.ejmg.2014.02.004.

9. Rumsey N, Harcourt D. Body image and disfigurement: issues and interventions. Body Image 2004; 1: 83-97. doi:10.1016/S1740-1445(03)00005.

10. Thompson A, Kent G. Adjusting to disfigurement: processes involved in dealing with being visibly different. Clin Psychol Rev 2001; 21:5, 663-82. [Acceso 10 de enero de 2018]. Disponible en: http://www.ncbi.nlm.nih.gov/pubmed/11434225. 
11. Wolkenstein P, Zeller J, Revuz J, Ecosse E, Leplège A. Visibility of neurofibromatosis 1 and psychiatric morbidity. Arch Dermatol 2003; 139:103-4. doi: 10.1001/ archderm.139.1.103.

12. Mouridsen SE, Sørensen SA. Psychological aspects of von Recklinghausen neurofibromatosis (NF1). J Med Genet 1995; 32:921-4. Disponible en: http://www.ncbi. nlm.nih.gov/pubmed/8825915.

13. Merker VL, Bredella MA, Cai W, Kassarjian A, Harris GJ, Muzikansky A, et al. Relationship between whole-body tumor burden, clinical phenotype, and quality of life in patients with neurofibromatosis. Am J Med Genet A 2014; 164A: 1431-7. doi:10.1002/ ajmg.a.36466.

14. Vranceanu A-M, Merker VL, Park E, Plotkin SR. Quality of life among adult patients with neurofibromatosis 1 , neurofibromatosis 2 and schwannomatosis: a systematic review of the literature. J Neurooncol 2013; 114: 257-62. doi:10.1007/s11060-014-1522-2.

15. Barke J, Harcourt D, Coad J. "It”s like a bag of pick and mix - you don't know what you are going to get': young people's experience of neurofibromatosis Type 1. J Adv Nurs 2014; 70: 1594-603. doi: 10.1111/jan.12319.

16. Hummelvoll G, Antonsen KM. Young adults' experience of living with neurofibromatosis type 1. J Genet Couns 2013; 22: 188-99. doi:10.1007/s10897-012-9527-5.

17. Smith KB, Wang DL, Plotkin SR, Park ER. Appearance concerns among women with neurofibromatosis: Examining sexual/bodily and social self-consciousness. Psychooncology 2013; 22: 2711-9. doi: 10.1002/pon.3350.

18. Kodra Y, Giustini S, Divona L, Porciello R, Calvieri S, Wolkenstein P, et al. Healthrelated quality of life in patients with neurofibromatosis type 1. A survey of 129 Italian patients. Dermatology 2009; 218: 215-20. doi.org/10.1159/000187594.

19. Bicudo NP, de Menezes Neto BF, da Silva de Avó LR, Germano CMR, Melo DG. Quality of life in adults with Neurofibromatosis 1 in Brazil. J Genet Couns 2016; 25: 1063-74. doi:10.1007/s10897-016-9939-8.

20. Descheemaeker M-J, Plasschaert E, Frijns J-P, Legius E. Neuropsychological profile in adults with neurofibromatosis type 1 compared to a control group. J Intellect Disabil Res 2013; 57: 874-86. doi: 10.1111/j.1365-2788.2012.01648.x.

21. Wang DL, Smith KB, Esparza S, Leigh FA, Muzikansky A, Park ER, et al. Emotional functioning of patients with neurofibromatosis tumor suppressor syndrome. Genet Med 2012; 14: 977-82. doi: 10.1038/gim.2012.85.

22. Cohen JS, Levy HP, Sloan J, Dariotis J, Biesecker BB. Depression among adults with neurofibromatosis type 1: Prevalence and impact on quality of life. Clin Genet 2015; 88: 425-30. doi: 10.1111/cge.12551

23. Vranceanu A-M, Merker VL, Plotkin SR, Park ER. The relaxation response resiliency program (3RP) in patients with neurofibromatosis 1, neurofibromatosis 2, and schwannomatosis: Results from a pilot study. J Neurooncol 2014; 120: 103-9. doi. org/10.1007/s11060-014-1522-2.

24. Pride NA, Crawford H, Payne JM, North KN. Social functioning in adults with neurofibromatosis type 1. Res Dev Disabil 2013; 34: 3393-9. doi: 10.1016/j. ridd.2013.07.011.

25. Pride NA, Korgaonkar MS, Barton B, Payne JM, Vucic S, North KN. The genetic and neuroanatomical basis of social dysfunction: Lessons from neurofibromatosis type 1 . Hum Brain Mapp 2014; 35: 2372-82. doi.org/10.1002/hbm.22334. 
26. Ejerskov C, Lasgaard M, Østergaard JR. Teenagers and young adults with neurofibromatosis type 1 are more likely to experience loneliness than siblings without the illness. Acta Paediatr 2015; 104: 604-9. doi: 10.1111/apa.12946.

27. Rozario S. Growing up and living with neurofibromatosis1 (NF1): a British Bangladeshi case-study. J Genet Couns 2007; 16: 551-9. doi: 10.1007/s10897-007-9097-0. 